\title{
Kinetic analysis of solid-state reactions: Precision of the activation energy calculated by integral methods
}

L.A. PÉREZ-MAQUEDA*, P.E. SÁNCHEZ-JimÉNEZ AND J.M. CRIADO

Instituto de Ciencia de Materiales de Sevilla. C.S.I.C.-Universidad de Sevilla. Avd. Americo Vespucio 41092 Sevilla. Spain

\begin{abstract}
The integral methods are extensively used for the kinetic analysis of solid-state reactions. The Arrhenius integral function $[p(x)]$ does not have an exact analytical solution. Thus, different approaches, accomplishing the condition that $\ln g(\alpha)$ is a linear function of either $1 / T$ or a predetermined function of $T$, have been proposed for this integral to determine the activation energy from a linear plot of the logarithm of $g(\alpha)$ versus some function of $T$. The first approach was proposed by Van Krevelen and after that, a number of authors developed new approaches, very often with the scope of increasing the precision of the Arrhenius integral as checked from the standard deviation of the $p\left(x_{a}\right)$ function determined from these approximation with regards to the true value of the $p(x)$ function. Besides this method, those proposed by Doyle, Horowitz and Metzger, Coats and Redfern, MacCallum and Tanner and Gyulay and Greenhow are very popular for determining activation energies. In fact, we have found more than 4500 citations (1300 in the last five years) for the papers were these methods were proposed. However, a systematic analysis of the errors involved in the determination of the activation energy from these methods is still missing. A comparative study of the precision of the activation energy as a function of $x$ and $T$ computed from the different integral methods has been carried out.
\end{abstract}

Keywords: Arrhenius integral, integral methods, solid state reactions, errors in activation energy. 


\section{INTRODUCTION}

Thermally stimulated solid-state reactions, such as decompositions, solid-solid reactions, crystallizations, etc, are, in general, heterogeneous processes. The reaction rate of such processes can be kinetically described, when it takes place under conditions far from equilibrium, by the following expression: ${ }^{1}$

$$
\frac{d \alpha}{d t}=f(T) f(\alpha)
$$

where $t$ is the time and $\alpha$ is the extent of reaction ranging from 0 before the process starts to 1 when it is over. Thus, the left hand side term in eq. (1) is the reaction rate. The right hand side term in eq. (1) consists of two terms, i.e. $f(T)$ and $f(\alpha)$, being $f(T)$ a function that describes the dependence of the reaction rate with the temperature $(T)$. Usually, this dependence is described by the Arrhenius equation:

$$
f(T)=A e^{-E / R T}
$$

being $\mathrm{A}$ the preexponential factor of Arrhenius, $\mathrm{E}$ the activation energy and $\mathrm{R}$ the gas constant. Additionally, $f(\alpha)$ is a term that describes the dependence of the reaction rate with the mechanism of the process. Different functions have been proposed in literature for describing the kinetic mechanism of the solid-state reactions. These mechanisms are proposed considering different geometrical assumptions for the shape of the material particles (spherical, cylindrical, planar) and driving forces (interface growth, diffusion, nucleation and growth of nuclei). Some of the most common equations proposed for these reactions are included in Table 1.

The most common heating profile used for studying solid-state reaction is the linear heating program. Under these experimental conditions, $T$ changes in a wide range of values and a entire $\alpha-T$ curve is recorded in a single experiment. For linear heating rate conditions eq. (1) can be written 


$$
\frac{d \alpha}{d T}=\frac{A}{\beta} e^{-E / R T} f(\alpha)
$$

being $\beta$ the heating rate.

Many of the experimental methods used to perform kinetic analysis of solid-state reactions are based in the measurement of the evolution of an integral magnitude, i.e. proportional to the extent of reaction, such as mass loss, released gas, amount of contraction, as a function of temperature. To perform the evaluation of such experimental data, it is necessary either to numerically differentiate the experimental data or to integrate eq. (3):

$$
g(\alpha)=\frac{A E}{\beta R} \int_{x}^{\infty} \frac{e^{-x}}{x^{2}} d x=\frac{A E}{\beta R} p(x)
$$

being $x=E / R T$. This expression can be written in the logarithmic form:

$$
\ln (g(\alpha))=\ln \frac{A E}{\beta R}+\ln p(x)
$$

Under linear heating rate program, eqs. 4 and 5 do not have an exact analytical solution to $p(x)$ and, therefore, the solution cannot be expressed in a closed form. ${ }^{2}$ Although, other $T$ - $t$ profiles, such as parabolic or hyperbolic programs, yield to analytical solutions to the Arrhenius integral, they are very seldom used. Thus, several approximated equations have been proposed for $p(x)$ under linear heating program.

The approximations for $p(x)$ most commonly used in the determination of the activation energy are those proposed by Coats and Redfern, ${ }^{3,4}$ Doyle, ${ }^{5-7}$ Horowitz and Metzger, ${ }^{8}$ MacCallum and Tanner, ${ }^{9,10}$ Gyulai and Greenhow, ${ }^{11,12}$ and Van Krevelen. ${ }^{13}$ All these approximations have been obtained either by simplifications of the series expressions or in an empirical way. For a given kinetic model, the resulting equations lead to a linear correlation where the kinetic activation energy is easy obtained from the slope. The number of publications where these integral methods are used for 
determining activation energies is vast. Thus, about 4500 citations can be found in the literature for the original papers ${ }^{3-13}$ where these equations are proposed. Besides, the popularity of these integral methods has not decreased, as indicated by their more than 1300 citations just in the last years, i.e. 2000-2004. In these last five years, the approach with more citations has been that of Coats and Redfern ${ }^{3,4}$ with about 590 citations, followed by those of Horowitz and Metzner ${ }^{8}$ and Doyle ${ }^{5-7}$ with 230 and 102 citations, respectively (information on the number of citations have been obtained from ISI Web of Science data base). Nevertheless, independently of the approximation used, every $g(\alpha)$ leads to a high linear correlation coefficient and, therefore, it is not possible to discriminate the kinetic model from a single experimental curve. Additionally, the resulting activation energy values are very much dependant on the $g(\alpha)$ function assumed for the analysis (these limitations are extended not only to integral methods but also to any procedure that uses a single linear heating rate curve ${ }^{14,15}$ ). Thus, in principle, the integral methods should be only used under the two following circumstances: (i) when the kinetic model is already known for obtaining the activation energy or (ii) when the activation energy is known for determining the kinetic model. Nevertheless, a new question arises about the precision of the activation energy values determined by these popular integral methods because, as mentioned above, they are based in approximations to the $p(x)$ function and their precision for the estimation of the kinetic parameters are still in doubt, thus some authors have claimed that these methods are imprecise. ${ }^{16-19}$ Some studies have estimated the errors in the approximated $p(x)$ functions by comparing the resulting values with those calculated by numerical integration, concluding that the errors are quite large. Fig. 1 shows as a way of example the evolution of the relative error of the Coats and Redfern approximation for the estimation of the $p(x)$ function versus $x$. This figure indicates that the error decreases 
with $x$, being significantly large for values of $x$ commonly found in literature for solidstate reactions. These findings have been used as an argument for invalidating these approximated equations in the estimation of the kinetic parameters. Nevertheless, the aim of the aforementioned approximations is the determination of the activation energy and not the accurate computation of $p(x)$. Taking into account that the integral methods are so widely extended and that there is some controversy in their precision, it would be of interest to estimate the precision of such methods for the determination of the activation energy. The aim of the present paper is to perform a comparative study of the precision of the most extensively used approximations to $p(x)$ in the determination of the activation energy.

\section{ERRORS IN THE ACTIVATION ENERGY}

\subsection{Coats and Redfern method.}

The Coats and Redfern ${ }^{3,4}$ approach to the Arrhenius integral is the following:

$$
p\left(x_{a}\right)=\frac{-e^{-x_{a}}}{x_{a}}\left(1-\frac{2}{x_{a}}\right)
$$

the subscript $a$ stands for approximated. In general, the expression more commonly used is the simplified form:

$$
p\left(x_{a}\right)=\frac{e^{-x_{a}}}{x_{a}^{2}}
$$

This approach is named sometimes in literature as Fisher approach. ${ }^{20}$ By introducing eq.

(7) into eq. (4), it follows

$$
g(\alpha)=\frac{A_{a} R}{E_{a} \beta} T^{2} e^{-E_{a} / R T}
$$

By taking natural logarithms, eq. (8) results 


$$
\ln (g(\alpha))=\ln \frac{A_{a} R T^{2}}{E_{a} \beta}-\frac{E_{a}}{R T}
$$

Thus, the activation energy could be easily obtained from the slope of the line resulting of plotting $\ln (g(\alpha))-2 \ln (T)$ versus $1 / T$.

The relative error $\varepsilon$ of the activation energy $\left(E_{a}\right)$ calculated by the Coats and Redfern equation can be defined by the following equation:

$$
\varepsilon \%=\frac{E_{a}-E}{E} 100=\left(\frac{\frac{E_{a}}{R}}{\frac{E}{R}}-1\right) 100
$$

By differentiating eq. (9):

$$
\frac{\partial \ln g(\alpha)}{\partial 1 / T}=\frac{-E_{a}}{R}-2 T
$$

and by differentiating eq. (5):

$$
\frac{\partial \ln g(\alpha)}{\partial 1 / T}=\frac{\partial \ln (p(x))}{\partial 1 / T}=\frac{E}{R} \frac{\partial \ln (p(x))}{\partial x}
$$

Thus, from eqs (11) and (12), it follows

$$
\frac{\frac{E_{a}}{R}}{\frac{E}{R}}=\frac{-\partial \ln (p(x))}{\partial x}-\frac{2}{x}
$$

that substituting in eq. (10) leads to

$$
\varepsilon \%=\left(-\frac{\partial \ln (p(x))}{\partial x}-\frac{2}{x}-1\right) 100
$$

This equation indicates that the values of $\varepsilon \%$ depend on $x=(E / R T)$, and, therefore, on the value of the activation energy and of the range of temperature of the process. The values of $\varepsilon \%$ have been computed by means of the Mathcad software by numerical integration of the $p(x)$ function using a tolerance (precision in the calculus) of $10^{-5}$. The resulting $\varepsilon \%$ values as a function of the parameter $x$ are included in Table 2 . The values 
included in Table 2 illustrate that there is a significant influence of $x$ in the precision of the calculated activation energy values. Thus, $\varepsilon \%$ ranges from almost $-20 \%$ for $x=2$ to less than $-1 \%$ for $x$ values larger than 20 ; in the limit, for $x=\infty$, the error is cero.

\subsection{Doyle method.}

The Doyle approach to the Arrhenius integral is the following: $:^{5-7}$

$$
\log \left(p\left(x_{a}\right)\right)=-2.315-0.4567 x_{a}
$$

From eq. (15) and eq. (5), it follows

$$
\log (g(\alpha))=\log \frac{A_{a} E}{\beta R}-2.315-0.4567 \frac{E}{R T}
$$

Thus, the activation energy can be obtained from the slope of the line resulting from plotting the left hand side of eq. (16) as a function of 1/T:

$$
\frac{\partial \log (g(\alpha))}{\partial 1 / T}=-0.4567 \frac{E_{a}}{R}
$$

The relative error $\varepsilon \%$ (eq. (10)) for the activation energy obtained by the Doyle method can be obtained from eqs. (12) and (17):

$$
\varepsilon \%=\left(\frac{-0.4343}{0.4567}\left(\frac{\partial \ln (p(x))}{\partial x}\right)-1\right) 100
$$

The values of $\varepsilon \%$ have been computed by the same procedure as described in the latter section and the resulting error values are included in Table 2.

\subsection{Horowitz and Metzger method.}

The integer equation after assuming the Horowitz and Metzger approach ${ }^{8}$ to the $p(x)$ function is the following: 


$$
\ln (g(\alpha))=\ln \frac{A_{a} E_{a}}{\beta R}-5.33-\frac{1.052}{T_{s}}+\frac{1.052 E_{a} \theta}{R T_{s}^{2}}
$$

where $\theta$ is a characteristic temperature such that $\theta=T-T_{s}$, being $T_{s}$ an arbitrary reference temperature. From eq. (19), it is clear that the activation energy is obtained from the slope of the line resulting of plotting the left hand side of eq. (19) versus $\theta$, or versus $T$ that yields the same slope:

$$
\frac{\partial \ln (g(\alpha))}{\partial T}=\frac{E_{a}}{R T_{s}^{2}}
$$

From eqs. (12) and (20), the relative error $\varepsilon \%$ (eq. (10)) in the activation energy obtained by the Horowitz and Metzger ${ }^{8}$ results:

$$
\varepsilon \%=\left(-\frac{\partial \ln (p(x))}{d x}-1\right) 100
$$

Table 2 includes the errors estimated by eq. (21) for the activation energy calculated by the Horowitz and Metzger approach. ${ }^{8}$

\subsection{Van Krevelen method}

Considering the Van Krevelen et al approximation ${ }^{13}$ to the exponential integral of Arrhenius, eq. (5) has the logarithmic form:

$$
\ln (g(\alpha))=\ln \left[\frac{A_{a}}{\beta E_{a}}\left(\frac{0.368}{T_{\max }}\right)^{\frac{E_{a}}{R T_{\max }}}\left(T_{\max }+1\right)\right]+\frac{E_{a}}{R\left(T_{\max }+1\right)} \ln T
$$

where $T_{\max }$ is the temperature at the maximum thermogravimetric rate. The activation energy is determined from the slope of the line resulting from the plot of $\ln (g(\alpha))$ as a function of $\ln T$ :

$$
\frac{\partial \ln (g(\alpha))}{\partial \ln T}=\frac{E_{a}}{R\left(T_{\max }+1\right)}
$$


Thus, the Van Krevelen et al method, ${ }^{13}$ even though it is a integer equation, for the determination of the activation energy, it requires of the differential experimental curve to obtain the $T_{\max }$ value for eq. (23).

As in the previous sections, the relative error can be calculated from eq. (12) and (23), resulting:

$$
\varepsilon^{\%}=\left(-\left(\frac{\partial \ln (p(x))}{\partial x}\right)+\frac{1}{x}-1\right) 100
$$

The resulting values for the error are included in Table 2.

\subsection{MacCallum and Tanner method}

The decimal logarithmic form of the integer equation (eq. (5)) using the approach proposed by MacCallum and Tanner ${ }^{9,10}$ to the $p(x)$ function results:

$$
\log (g(\alpha))=\log \frac{A_{a} E_{a}}{\beta R}-0.4828 E_{a}^{0.4351}-\frac{449+217 E_{a}}{T}
$$

Thus, the activation energy can be calculated from the slope of the line resulting from the plot of $\log (g(\alpha))$ as a function of $1 / \mathrm{T}$ :

$$
\frac{\partial \log (g(\alpha))}{\partial(1 / T)}=-449-217 E_{a}
$$

The relative error $\varepsilon \%$ (eq. (10)) of the activation energy can be calculated from eqs. (12) y (26):

$$
\varepsilon \%=\left(\frac{-0.4343}{217 R} \cdot \frac{\partial \ln (p(x))}{\partial x}-\frac{449}{217 x R T}-1\right) 100
$$

In this case the error depends both on $x$ and $T$. Table 3 includes the errors in the activation energy as estimated by means of eq. (27). 


\subsection{Gyulai and Greenhow method}

The decimal logarithmic form of the integer equation eq. for the approach of Gyulai and Greenhow ${ }^{11,12}$ can be written as follows:

$$
\log (g(\alpha))=\log \frac{A_{a} E_{a}}{\beta R}+\log \frac{R}{E_{a}}-3.542051-0.915784 \log E_{a}-269.645 \frac{E_{a}^{.9583}}{T}
$$

The activation energy can be calculated from the slope of the line resulting from the plot of $\log (g(\alpha))$ as a function of $1 / \mathrm{T}$ :

$$
\frac{\partial \log (g(\alpha))}{\partial(1 / T)}=-269.654 E_{a}^{0.9583}
$$

As in the previous sections, the relative error can be calculated from eq. (12) and (29), resulting:

$$
\varepsilon \%=\left(\frac{-1}{x R T}\left(\frac{0.4343 x T}{269.654} \cdot \frac{\partial \ln (p(x))}{\partial x}\right)^{1 / 0.9583}-1\right) 100
$$

This expression has some similarities with that for the errors in the activation energy of MacCallum y Tanner (eq. (24)). Thus, here the $\varepsilon \%$ also depends on $x$ and $T$ (Table 4).

\section{CHECKING OF THE COMPUTED ERRORS WITH THEORETICAL AND EXPERIMENTAL CURVES.}

To check the validity of the errors calculated in the previous section, a set of experimental curves have been simulated and analyzed by the integral methods using the different approaches analyzed here. Two different curves have been simulated assuming two different kinetic models, kinetic parameters, and linear heating rate conditions. The simulated curves have been computed by solving the system of two differential equations constituted by eq. (1) and $\partial T / \partial t=\beta$ by means of the RungeKutta method using the mathcad software and a tolerance (precision in the calculus) of 
$10^{-5}$. The first curve (Fig. 2a) has been simulated for $\beta=10 \mathrm{~K} \mathrm{~min}^{-1}$, an A2 kinetic model and the following kinetic parameters: $E=35 \mathrm{~kJ} \mathrm{~mol}^{-1}$ and $A=10 \mathrm{~min}^{-1}$. The second curve (Fig. 2b) has been computed for $\beta=1 \mathrm{~K} \mathrm{~min}^{-1}$, an F1 kinetic model and the following kinetic parameters: $E=100 \mathrm{~kJ} \mathrm{~mol}^{-1}$ and $A=10^{8} \mathrm{~min}^{-1}$. The average values of $x$ for these two curves are 5 and 20 for the first (Fig. 2a) and second (Fig. 2b) curves, respectively. These curves have been analyzed by means of the integer method using the different approaches analyzed above. The resulting values of activation energy and the corresponding errors are given in Table 5. The errors included in Table 5 for the different approaches are consistent with those reported in Tables 2-4. The small differences between the errors in Table 5 and Tables 2-4 are due to the fact that the errors in Tables 2-4 have been calculated at constant values of $x$, while those reported in Table 5 do not correspond to a single $x$ value but to a range of $x$ values because the temperature varies in a $\alpha$-T curve while the activation energy is constant.

For checking the precision of the method with experimental data, a thermogravimetric (TG) curve was recorded for the decomposition of $\mathrm{BaCO}_{3}$ under high vacuum conditions. This is a very stable compound with low equilibrium pressure $\left(\sim 3.510^{-3}\right.$ torr $)$ in the temperature range $(1000 \mathrm{~K})$ at which the reaction takes place. Therefore, the decomposition conditions should be properly controlled for maintaining the partial pressure of $\mathrm{CO}_{2}$ far away from the equilibrium pressure. ${ }^{21}$ Such condition was fulfilled by using small amount of sample $(10 \mathrm{mg})$, low heating rate $\left(0.2 \mathrm{~K} \mathrm{~min}^{-1}\right)$, and performing the experiment under high vacuum (the balance was connected to a vacuum device that reduced the total pressure to $310^{-5}$ torr). Additionally, during the TG experiment, the partial pressure of $\mathrm{CO}_{2}$ was recorded with a quadrupole-massspectrometer to make sure that $\mathrm{CO}_{2}$ partial pressure was far away from equilibrium pressure. In fact, the $\mathrm{CO}_{2}$ pressure did not increase during the thermal decomposition 
above $110^{-6}$ torr. The thermogravimetric curve obtained under the conditions previously described is included in Fig. 3. Table 6 includes the activation energies and correlation factors resulting from the analysis of the experimental data (Fig. 3) by means of the integer methods analyzed here. It is worth noting that the correlation factors are very high for all the approximated equations. Additionally, the experimental integral curve was numerically differentiated with the Microcal Origin software and analyzed the logarithmic form of the equation resulting from eqs. (1) and (2): $:^{14,15,22,23}$

$$
\ln \frac{d \alpha}{d t}-\ln (f(\alpha))=\ln (A)-\frac{E}{R T}
$$

The activation energy can be directly obtained from the slope of the line resulting from the plot of the left hand side of eq. (26) as a function of $1 / T$. The resulting activation energy and correlation factor has been also included in Table 6. Using the activation energy calculated from the differential equation (eq. 26) as accurate value (because no approximation is involved in the method), we have calculated the errors of the activation energies determined by the different integer methods (Table 6). The resulting errors are in the range of the expected ones (Tables 2-4) for the value of $x=25$ corresponding to this experiment, the small deviations observed are due to experimental errors and to the fact that $x$, as also mentioned before, is not constant during the entire process.

\section{SUMMARY}

In this paper we have calculated the errors in the activation energy determined by the extensively used integral methods. These methods are based in approximated equations of the Arrhenius integral that lead to linear relation between the logarithmic form of $g(\alpha)$ and a function of the temperature from whose slope is determined the activation energy. The values of activation energy obtained by the integral method are 
subjected to some impressions because these methods are based on approximations. It is worth to note that, as mentioned above, every kinetic model lead a good linear correlation and, therefore, from a single linear heating rate program curve it is not possible to determine the activation energy of the process unless the kinetic model is known. The scope of the paper is the quantification of the errors in the activation energy determined by integral methods when the kinetic model is known. The quantification of those errors has not been directly related to the precision of the approximated $\mathrm{p}(\mathrm{x})$ function for evaluating the temperature integral, because the application of these proposed approximations is the determination of the activation energy and not the calculation of the temperature integral. The error analyses have shown that for all the approaches analyzed here, the relative errors very much depend on $x$; that is, on $E$ and on the average temperature of the process. Additionally, this average temperature of the process depends on the value of $E, A$ and the kinetic model followed by the reaction. Thus, the error of the activation energy calculated by the integral method is influenced by the kinetic parameters of the process. In general, small values of $x$ due to small values of $E$ and/or high values of temperature yield relatively high errors and the integral methods are not appropriate. On the other hand, for high values of $x$, the errors are quite small. It is worth noting that the approach that leads to the minimum error in the activation energy is that of Coats and Redfern. Finally, the calculated error values have been checked with those obtained of the analysis of simulated and experimental curves showing an excellent agreement. 


\section{REFERENCES}

(1) Criado, J. M.; Pérez Maqueda, L. A., In Sample Controlled Thermal Analysis: Origin, Goals, Multiple Forms, Applications and Future; Sorensen, O. T., Rouquerol, J., Eds.; Kluwer: Dordrecht, 2003; Vol. 3, p 55.

(2) Pérez-Maqueda, L. A.; Criado, J. M. J. Therm. Anal. Calorim. 2000, 60, 909.

(3) Coats, A. W.; Redfern, J. P. Nature 1964, 201, 68.

(4) Coats, A. W.; Redfern, J. P. Journal of Polymer Science Part B-Polymer Letters 1965, 3, 917.

(5) Doyle, C. D. Analytical Chemistry 1961, 33, 77.

(6) Doyle, C. D. Journal of Applied Polymer Science 1961, 5, 285.

(7) Doyle, C. D. Nature 1965, 207, 290.

(8) Horowitz, H. H.; Metzger, G. Anal. Chem. 1963, 35, 1464.

(9) MacCallum, J. R.; Tanner, J. European Polymer Journal 1970, 6, 1033.

(10) MacCallum, J. R.; Tanner, J. European Polymer Journal 1970, 6, 907.

(11) Gyulai, G.; Greenhow, E. J. Thermochimica Acta 1973, 6, 239.

(12) Gyulai, G.; Greenhow, E. J. Journal of Thermal Analysis 1974, 6, 279.

(13) van Krevelen, D. W.; van Heerden, C.; Huntjens, F. J. Fuel 1951, 30, 253.

(14) Perez-Maqueda, L. A.; Criado, J. M.; Gotor, F. J.; Malek, J. Journal of Physical Chemistry A 2002, 106, 2862.

(15) Perez-Maqueda, L. A.; Criado, J. M.; Malek, J. Journal of Non-Crystalline Solids 2003, 320, 84.

(16) Heal, G. R. Thermochimica Acta 1999, 341, 69.

(17) Heal, G. R. Instrumentation Science \& Technology 1999, 27, 367. 
(18) Flynn, J. H. Thermochimica Acta 1997, 300, 83.

(19) Flynn, J. H. Thermochimica Acta 1992, 203, 519.

(20) Fisher, P. E.; Chon, S. J.; S.S., G. Ind. Eng. Chem. Res. 1987, 26, 1037.

(21) Pérez-Maqueda, L. A.; Criado, J. M.; Gotor, F. I. International Journal of Chemical Kinetics 2002, 34, 184.

(22) Friedman, H. L. J Polym Sci C 1965, 6, 183.

(23) Criado, J. M.; Perez-Maqueda, L. A.; Gotor, F. J.; Malek, J.; Koga, N. Journal of Thermal Analysis and Calorimetry 2003, 72, 901. 
TABLE 1. $f(\alpha)$ and $g(\alpha)$ kinetic functions

\begin{tabular}{|c|c|c|c|}
\hline Mechanism & Symbol & $f(\alpha)$ & $g(\alpha)$ \\
\hline $\begin{array}{l}\text { Phase boundary controlled reaction } \\
\text { (contracting area) }\end{array}$ & $\mathrm{R} 2$ & $(1-\alpha)^{1 / 2}$ & $2\left[1-(1-\alpha)^{1 / 2}\right]$ \\
\hline $\begin{array}{l}\text { Phase boundary controlled reaction } \\
\text { (contracting volume) }\end{array}$ & R3 & $(1-\alpha)^{2 / 3}$ & $3\left[1-(1-\alpha)^{1 / 3}\right]$ \\
\hline $\begin{array}{l}\text { Random nucleation followed by an } \\
\text { instantaneous growth of nuclei. } \\
\text { (Avrami-Erofeev eqn. } n=1 \text { ) }\end{array}$ & ${ }^{1} \mathrm{~F} 1$ & $(1-\alpha)$ & $-\ln (1-\alpha)$ \\
\hline $\begin{array}{l}\text { Random nucleation and growth of } \\
\text { nuclei through different nucleation } \\
\text { and nucleus growth models. (Avrami- } \\
\text { Erofeev eqn.) }\end{array}$ & An & $n(1-\alpha)[-\ln (1-\alpha)]^{1-1 / n}$ & {$[-\ln (1-\alpha)]^{1 / n}$} \\
\hline Two-dimensional diffusion & D2 & $1 /[-\ln (1-\alpha)]$ & $(1-\alpha) \ln (1-\alpha)+\alpha$ \\
\hline $\begin{array}{l}\text { Three-dimensional diffusion } \\
\text { (Jander equation) }\end{array}$ & D3 & $\frac{3(1-\alpha)^{2 / 3}}{2\left[1-(1-\alpha)^{1 / 3}\right]}$ & {$\left[1-(1-\alpha)^{1 / 3}\right]^{2}$} \\
\hline $\begin{array}{l}\text { Three-dimensional diffusion } \\
\text { (Ginstling-Brounshtein equation) }\end{array}$ & D4 & $\frac{3}{2\left[(1-\alpha)^{-1 / 3}-1\right]}$ & $(1-2 \alpha / 3)-(1-\alpha)^{2 / 3}$ \\
\hline
\end{tabular}

${ }^{1}$ This equation represents an Avrami-Erofeev kinetic model with $n=1$ instead of a first order reaction.

The symbol A1 would be more proper. 
TABLE 2. Values of the relative error $(\varepsilon \%)$ for the activation energy calculated by means of the Coats and Redfern, Doyle, Horowitz and Metzger, and Van Krevelen et al. equations as a function of the parameter $x(E / R T)$.

\begin{tabular}{|r|c|c|c|c|}
\hline \multicolumn{1}{|c|}{$\boldsymbol{x}$} & $\begin{array}{c}\text { Coats and } \\
\text { Redfern }\end{array}$ & Doyle & $\begin{array}{c}\text { Horowitz and } \\
\text { Metzger }\end{array}$ & Van Krevelen \\
\hline 2 & -19.72 & 71.43 & 80.28 & 30.28 \\
\hline 5 & -4.76 & 28.60 & 35.26 & 15.23 \\
\hline 10 & -1.47 & 12.72 & 18.53 & 8.53 \\
\hline 20 & -0.42 & 4.20 & 9.58 & 4.58 \\
\hline 30 & -0.20 & 1.25 & 6.47 & 3.13 \\
\hline 50 & -0.07 & -1.17 & 3.92 & 1.92 \\
\hline 100 & -0.02 & -3.02 & 1.98 & 0.98 \\
\hline$\infty$ & 0 & -4.90 & 0 & 0 \\
\hline
\end{tabular}

TABLE 3. Values of the relative error $(\varepsilon \%)$ for the activation energy calculated by means of the MacCallum and Tanner equation as a function of the parameter $x(E / R T)$ and the temperature $(T)$.

\begin{tabular}{|r|r|r|r|r|r|}
\hline $\boldsymbol{x}$ & $\mathbf{4 0 0}$ & $\mathbf{6 0 0}$ & $\mathbf{8 0 0}$ & $\mathbf{1 0 0 0}$ & $\mathbf{1 2 0 0}$ \\
\hline $\mathbf{2}$ & -48.55 & -5.14 & 16.56 & 29.59 & 38.27 \\
\hline $\mathbf{5}$ & -15.80 & 1.56 & 10.24 & 15.45 & 18.92 \\
\hline $\mathbf{1 0}$ & -6.59 & 2.09 & 6.43 & 9.03 & 10.77 \\
\hline $\mathbf{2 0}$ & -2.59 & 1.75 & 3.92 & 5.22 & 6.09 \\
\hline $\mathbf{3 0}$ & -1.38 & 1.51 & 2.96 & 3.83 & 4.40 \\
\hline $\mathbf{5 0}$ & -0.47 & 1.26 & 2.13 & 2.65 & 3.00 \\
\hline $\mathbf{1 0 0}$ & 0.17 & 1.04 & 1.47 & 1.73 & 1.91 \\
\hline $\boldsymbol{\infty}$ & 0.78 & 0.78 & 0.78 & 0.78 & 0.78 \\
\hline
\end{tabular}

TABLE 4. Values of the relative error $(\varepsilon \%)$ for the activation energy calculated by means of the Gyulai and Greenhow equation as a function of the parameter $x(E / R T)$ and the temperature $(T)$.

\begin{tabular}{|r|r|r|r|r|r|}
\hline $\boldsymbol{x}$ & $\mathbf{4 0 0}$ & $\mathbf{6 0 0}$ & $\mathbf{8 0 0}$ & $\mathbf{1 0 0 0}$ & $\mathbf{1 2 0 0}$ \\
\hline $\mathbf{2}$ & 50.25 & 52.93 & 54.86 & 56.37 & 57.61 \\
\hline $\mathbf{5}$ & 15.84 & 17.9 & 19.39 & 20.55 & 21.51 \\
\hline $\mathbf{1 0}$ & 4.04 & 5.89 & 7.23 & 8.27 & 9.14 \\
\hline $\mathbf{2 0}$ & -1.21 & 0.55 & 1.82 & 2.81 & 3.63 \\
\hline $\mathbf{3 0}$ & -2.43 & -0.69 & 0.56 & 1.54 & 2.35 \\
\hline $\mathbf{5 0}$ & -2.72 & -0.99 & 0.26 & 1.24 & 2.04 \\
\hline $\mathbf{1 0 0}$ & -1.70 & 0.05 & 1.31 & 2.30 & 3.12 \\
\hline$\infty$ & 18.90 & 18.90 & 18.90 & 18.90 & 18.90 \\
\hline
\end{tabular}


TABLE 5. Values of the activation energies $\left(E_{a}\right)$ and errors $(\varepsilon \%)$ obtained of the analysis of the simulated curves included in Figs $2 \mathrm{a}$ and $2 \mathrm{~b}$ by means of the different integral methods

\begin{tabular}{|l|l|l|l|l|}
\cline { 2 - 5 } \multicolumn{1}{c|}{} & \multicolumn{2}{c|}{$\begin{array}{c}\text { Simulated curve Fig. 2a } \\
(x \approx 5)^{*}\end{array}$} & \multicolumn{2}{c|}{$\begin{array}{c}\text { Simulated curve Fig. 2b } \\
(x \approx 20)^{*}\end{array}$} \\
\cline { 2 - 6 } \multicolumn{1}{c|}{} & $E_{a}\left(\mathrm{~kJ} \mathrm{~mol}^{-1}\right)$ & $\varepsilon \%$ & $E_{a}\left(\mathrm{~kJ} \mathrm{~mol}^{-1}\right)$ & $\varepsilon \%$ \\
\hline Coats and Redfern & 33.5 & -4.4 & 99.6 & -0.352 \\
\hline Doyle & 44.4 & 27.0 & 103.4 & 3.40 \\
\hline $\begin{array}{l}\text { Horowitz and } \\
\text { Metzger }\end{array}$ & 48.5 & 38.7 & 114.2 & 14.20 \\
\hline $\begin{array}{l}\text { MacCallum and } \\
\text { Tanner }\end{array}$ & 38.4 & 9.7 & 100.8 & 0.75 \\
\hline $\begin{array}{l}\text { Gyulai and } \\
\text { Greenhow }\end{array}$ & 41.7 & 19.1 & 100.6 & 0.63 \\
\hline Van Krevelen et al. & 41.9 & 19.8 & 106.9 & 6.93 \\
\hline
\end{tabular}

${ }^{*}$ The average value of $x$ has been obtained from $E / R T_{\alpha=0.5}$, where $T_{\alpha=0.5}$ is the temperature corresponding to $\alpha=0.5$.

TABLE 6. Values of the activation energies $\left(E_{a}\right)$ and errors $(\varepsilon \%)$ obtained of the analysis of the experimental results for the thermal decomposition of $\mathrm{BaCO}_{3}$ obtained under high vacuum (Fig. 3) by means of the different integral methods.

\begin{tabular}{|l|l|l|l|}
\hline Method & Correlation factor & $E_{a}$ & $\varepsilon^{\%}$ \\
\hline Coats and Redfern & 0.999 & 212.5 & -0.42 \\
\hline Doyle & 0.999 & 218.6 & 2.39 \\
\hline $\begin{array}{l}\text { MacCallum and } \\
\text { Tanner }\end{array}$ & 0.999 & 222.9 & 4.45 \\
\hline Gyulai Greenhow & 0.999 & 220.1 & 3.14 \\
\hline Horowitz Metzger & 0.999 & 230.3 & 7.92 \\
\hline Van Krevelen et al. & 0.999 & 222.6 & 4.31 \\
\hline Friedman & 0.999 & 213.4 & - \\
\hline
\end{tabular}

${ }^{*}$ The errors of the activation energies have been calculated using the activation energy determined by the Friedman equation as the accurate value (because no approximation is involved in the method). 


\section{Figure Captions}

Fig. 1. Evolution of the relative error of the Coats and Redfern approach for the estimation of the $p(x)$ function versus the value of $x$. The relative error has been defined by the expression: $\left(p_{a}(x)-p(x) / p(x)\right) \cdot 100$, being $p_{a}(x)$ the value obtained by the Coats and Redfern approximation and $p(x)$ the value obtained by numerical integration.

Fig. 2. Simulated curves (a) $\beta=10 \mathrm{~K} \mathrm{~min}^{-1}$, an $\mathrm{A} 2$ kinetic model and the following kinetic parameters: $E=35 \mathrm{~kJ} \mathrm{~mol}^{-1}$ and $A=10 \mathrm{~min}^{-1}$; and (b) $\beta=1 \mathrm{~K} \mathrm{~min}^{-1}$, an $\mathrm{F} 1$ kinetic model and the following kinetic parameters: $E=100 \mathrm{~kJ} \mathrm{~mol}^{-1}$ and $A=10^{8} \mathrm{~min}^{-1}$

Fig. 3. Experimental TG curve obtained for the $\mathrm{BaCO}_{3}$ under high vacuum at a heating rate of $0.2 \mathrm{~K} \mathrm{~min}^{-1}$. 


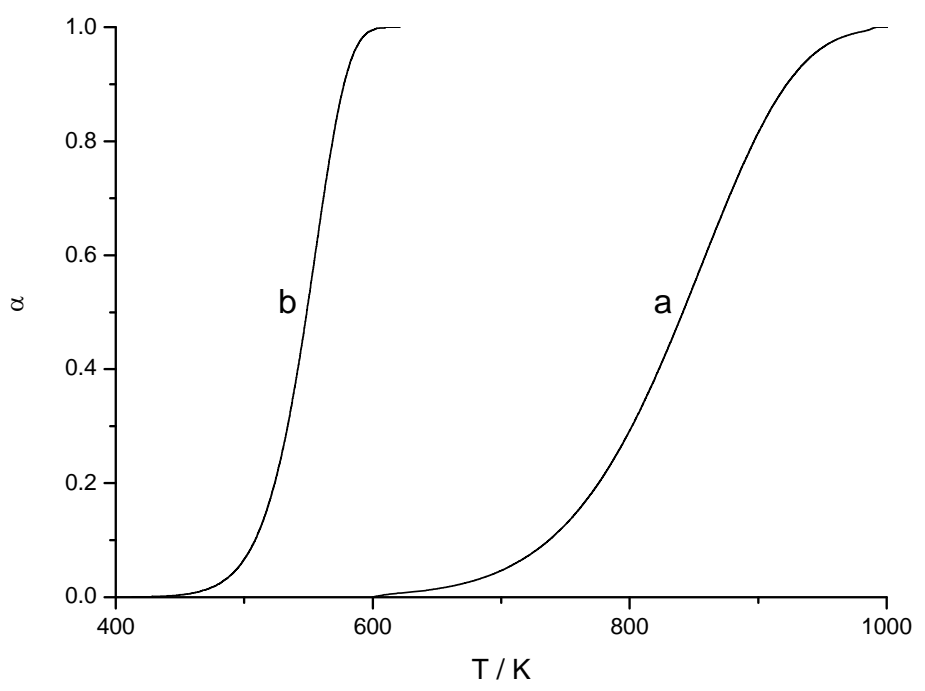

Fig. 1 


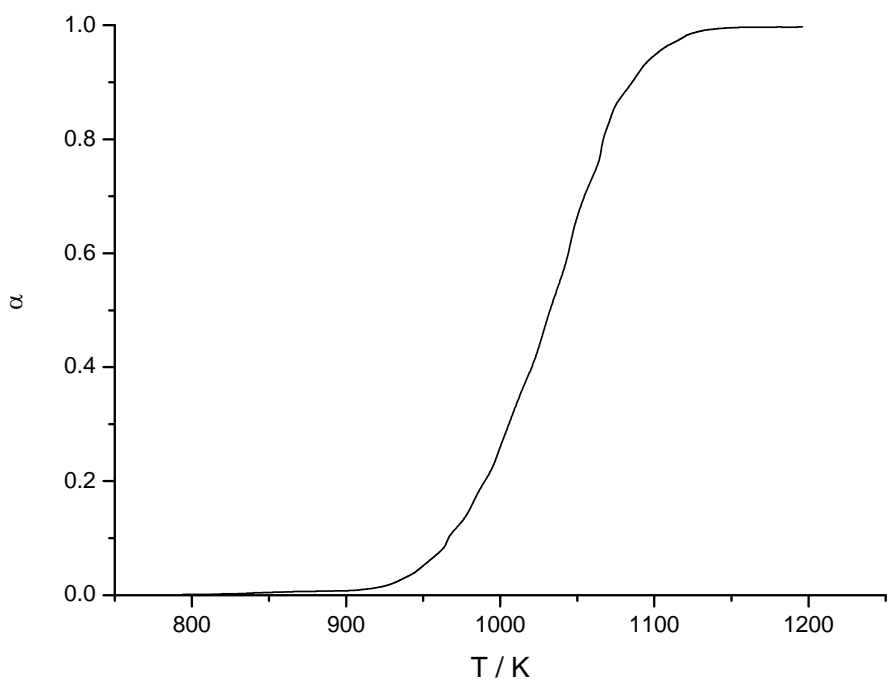

Fig. 2 